\title{
Observation of resonant states in negative refractive photonic crystals
}

\section{S. Romano}

silvia.romano@na.imm.cnr.it

\section{A. C. De Luca}

E. De Tommasi

\section{S. Cabrini}

I. Rendina

V. Mocella
CNR-IMM-Unità di Napoli, Via P. Castellino 111, 80131 Napoli, Italy

CNR-IBP, Via P. Castellino 111, 80131 Napoli, Italy

CNR-IMM-Unità di Napoli, Via P. Castellino 111, 80131 Napoli, Italy

Molecular Foundry, Lawrence Berkeley National Laboratory, Berkeley, USA

CNR-IMM-Unità di Napoli, Via P. Castellino 111, 80131 Napoli, Italy

CNR-IMM-Unità di Napoli, Via P. Castellino 111, 80131 Napoli, Italy

In this paper, experimental evidences about the resonance phenomena in a negative 2D photonic crystal are shown. Localized plasmon-like modes and guided mode resonances are detected in the reflectivity spectrum of a photonic crystal slab irradiated with out-of-plane incident radiation. The strong confinement of the radiation, in addition to the field enhancement, make photonic crystals a very appealing alternative to plasmonic substrates, avoiding the limits of absorption losses in metals.

[DOI: http://dx.doi.org/10.2971/jeos.2014.14006]

Keywords: Negative index, guided mode resonance, surface modes, photonic crystals

\section{INTRODUCTION}

Controlling and manipulating the light at nanoscale can play a key role in several physical phenomena in both fundamental and applied research. A large number of applications make use of light that is confined to small length scales, such as optical system processing, communication system and sensing. For example, label free nanoscale biosensor [1]-[7] can reveal advantages, compared to traditional sensing techniques, reducing the complexity, sensitivity and cost. In this landscape, surface plasmon resonance (SPR) sensors represent a key point, allowing the analysis of the kinetic of interactions between a biomolecule and its specific target with very high sensitivity and low detection limits, in real-time and without fluophore labeling. Among the applications of SPR in optical biosensing, a noticeable example is represented by surface enhanced Raman spectroscopy (SERS) [8]-[11], where local plasmon states are excited in proximity of metallic nano-particles by incident laser light with a consequent enhancement of the Raman signal (up to several order of magnitudes) from the analyzed molecules.

However, despite their remarkable performances, the resolution obtainable in resonance angle shift and the sensitivity of the SPR technique is limited by resonance width, which, in turn, depends on the large optical absorption induced by metallic layer $[12,13]$. Moreover, the local heating associated to such losses leads to high dissipations in the fluid medium carrying the analyte, inducing unwanted vortices in the car- rier flow and eventually damages in the biological material [14]. For these reasons alternative non-metallic loss-free substrates are particularly attracting.

Previous studies have shown that negative index photonic crystals (PhCs) can support surface waves similar to surface plasmons in metals, and the existence of these plasmonlike evanescent states has been experimentally observed $[15,16]$. Furthermore, the presence of guided mode resonances (GMRs) have been demonstrated in negative $\mathrm{PhC}$ slabs, generating sharp resonances in the reflection and transmission spectra when incident radiation is coupled with the modes of the structure [17]-[21]. While plasmon-like modes have the electromagnetic fields confined on the PhC surface, GMRs are confined into the structure. However, both phenomena can be directly connected with negative refractive index properties of PhCs, and can be described by Lorentz model.

In this paper, we present experimental measurements of these two separate resonant phenomena in a 2D PhC sample characterized by a negative effective permittivity, exploited by using two different optical configurations. Both the resonances are excited only at optical polarizations for which no band-gap is supported. We complete the analysis comparing the data carried out by the Lorentz model approach with the numerical re- 
sults obtained by both finite-difference time-domain (FDTD) method and rigorous coupled wave analysis (RCWA).

\section{THEORY}

Negative refracting behavior is an essential feature of a PhC. It provides unique optical properties, including unusual transmission and emission properties [22]-[25]. Recently, it has been demonstrated that the dielectric permittivity of $\mathrm{PhC}$ (non-metallic structures), in the frequency range where the refractive index is negative, is well described by a Lorentz model [26]:

$$
\varepsilon(\omega)=\frac{f \omega_{0}^{2}}{\omega_{0}^{2}-\omega^{2}+i \gamma \omega}+\varepsilon_{\infty}
$$

where $\varepsilon$ is the dielectric constant, $\omega_{0}$ the resonant frequency and $\gamma$ the damping constant. According to this model, the permittivity of a PhC can be negative at certain wavelength ranges and can support surface waves similar to the surface plasmon modes in metal.

Furthermore, this dielectric function model, Eq. (1), allows to obtain information about the origin of the coupling process between the external radiation and the photonic crystal structure in the GMRs phenomenon. In fact, the features of the reflection spectrum can be described by the usual Fresnel relation. In a three-layer system air/PhC/air, the reflectivity $\mathrm{R}$ for p-polarized light is given by:

$$
R=\left|r_{123}^{p}\right|^{2}=\left|\frac{r_{12}^{p}+r_{23}^{p} \exp \left(2 i k_{z 2} d\right)}{1+r_{21}^{p} r_{23}^{p} \exp \left(2 i k_{z 2} d\right)}\right|^{2}
$$

with the reflection coefficients given by:

$$
r_{i j}^{p}=\frac{\varepsilon_{j} k_{z i}-\varepsilon_{i} k_{z j}}{\varepsilon_{j} k_{z i}+\varepsilon_{i} k_{z j}}
$$

and the wave vector components along the $\mathrm{z}$ axis defined as :

$$
k_{z i}=\sqrt{\varepsilon_{i} \frac{\omega^{2}}{c^{2}}-\frac{\omega^{2}}{c^{2}} \sin ^{2} \theta}
$$

\section{EXPERIMENTAL DETAILS}

The 2D photonic crystal sample is obtained by a highprecision nanofabrication process with high-voltage electron beam lithography and a gas chopping inductively coupled plasma etching process which alternates an etching step using $\mathrm{SF}_{6}$, Ar with a passivation step using $\mathrm{CHF}_{3}$ and $\mathrm{CH}_{4}$. Starting with a Silicon-On-Insulator (SOI) wafer $(1.5 \mu \mathrm{m}$ silicon layer on top of $1 \mu \mathrm{m}$ oxide layer), we spin on ZEP 520, a positive electron beam (EB) resist at a thickness of $370 \mathrm{~nm}$. The resist is patterned using a Vistec VB300UHR EWF electron-beam lithography (EBL) System, and developed with n-amyl acetate. The EB patterned resist is used as a mask to etch the underlying silicon layer, down to the SOI, in an Oxford Plasmalab $100 \mathrm{ICP}=\mathrm{RIE}$ with resist mask. The $\mathrm{SF}_{6}$ chemistry provides the free radicals for isotropic Si etching, while $\mathrm{O}_{2}$ promotes the growth (at cryogenic temperatures) of a passivation film. This passivation film, with a generic temperature sensitive formula $\mathrm{Si}_{x} \mathrm{~F}_{y} \mathrm{O}_{z}$, protects the side walls of the etched structures and disappears after warming to room temperature leaving behind a clean surface. The etching was performed at $-120^{\circ} \mathrm{C}$. The resulting pattern is formed by cylindrical air holes and the device is characterized by a thickness of $0.7 \mu \mathrm{m}$ and an area of $1 \times 1 \mathrm{~mm}^{2}$, a lattice constant $\mathrm{a}=472 \mathrm{~nm}$, a hole radius $r=0.385 a$. The condition of negative index medium is guaranteed by the ratio $\mathrm{r} / \mathrm{a}=0.385$ [27]-[32]. In this way, the photonic crystal has an effective isotropic index $\mathrm{n}_{\text {eff }}=-1$ for the wavelength of $1.55 \mu \mathrm{m}$ corresponding to the isotropic circular shape of Equi-Frequency Surfaces. Consequently a nonlocal homogenization could be applied, as successfully demonstrated by Vynck et al. [33] in dielectric structure with the same refractive index contrast.

In this study, we experimentally analyze the resonance phenomena in the reflectivity spectrum of the PhC slab when an incident wave vector out of the slab plane impinges its top surface. For the plasmon-like resonance coupling, the usual Kretschmann configuration has been adopted while direct aircoupling have been used for the GMRs. For more details about the experimental condition, see [16]. The presence of the surface states in the 2D PhC slab has been detected by means of an evanescent-coupling method through a high refractive index prism. The experimental set-up is shown in Figure 1. A tunable CW diode laser (Ando AQ4321D; $\lambda=1520-1620 \mathrm{~nm}$, $P_{\max }=5 \mathrm{~mW}$ ) emits monochromatic light which is polarized parallel to the incident plane. The radiation is then focused by a long working distance 20x objective lens (Mitutoyo L03030105A) at the bottom face of a SFL11 prism (refractive index 1.74). A similar experimental setup has been used to detect the GMRs coupled into the PhC slab. In this case, light coupling occurs without mediation of a high index medium, removing the prism, because the modes are not confined below the light line. Also in this case the incident light is p-polarized and focused upon the top surface of the sample.

\section{RESULT AND DISCUSSION}

\subsection{Plasmon-like surface states}

The presence of the surface states in the 2D PhC has been characterized, in Kretschmann configuration, by measuring the reflection spectra from the PhC and varying the incidence angle $\theta$ in the range $57-63^{\circ}$ with a step of $1^{\circ}$, in the condition $\theta>\theta_{\text {crtitical }}$ (where $\theta_{\text {crtitical }}$ is the angle of incidence above which total internal reflection occurs and $\theta_{\text {critical }}=35.1^{\circ}$ ). When the coupling between the evanescent wave and the surface mode occurs, a typical resonance peak in the reflectivity spectrum is observable (Figure 2) together with a broad background, due to Fabry-Perot oscillations when light interacts with a uniform dielectric slab. This is a special case of resonant phenomenon between continuum and discrete states well described by Fano line-shape, and it can be observed under specific conditions in PhCs [34, 35]. 


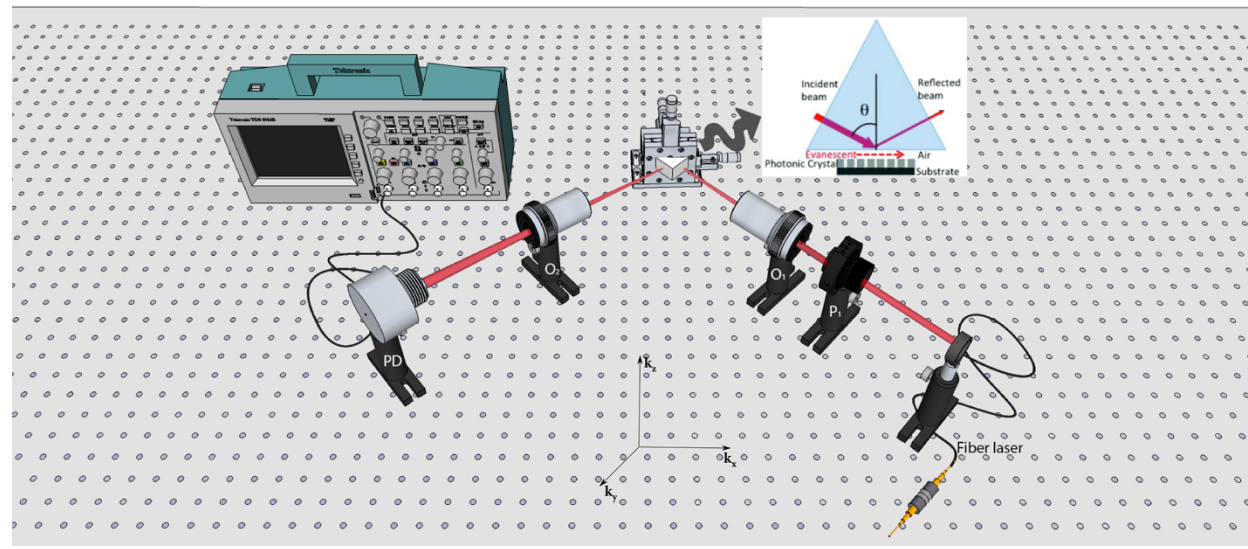

FIG. 1 Scheme of the experimental set-up used to excite the resonances. Abbreviations: 0: objective, PD: photodiode, P: polarizer. The same configuration is used for the GMRS detection, removing the prism.

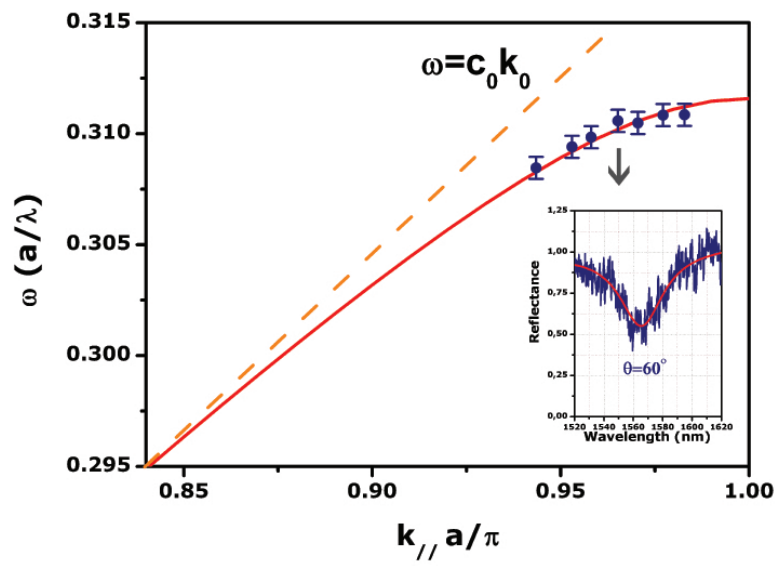

FIG. 2 Comparison between measured (blue dots) and simulated (red line) dispersion characteristic of the $2 \mathrm{D}$ PhC slab surface modes. The dashed line shows the light line at low frequency. The inset shows the experimental reflection signal at $\theta=60^{\circ}$ with the related best fit (red curve).

Therefore, all the data are well fitted by:

$$
R(\omega)=R_{d}+R_{g} \frac{\left(q \gamma+\omega-\omega_{0}\right)^{2}}{\left(\omega-\omega_{0}\right)^{2}+\gamma^{2}}
$$

where $\mathrm{R}_{d}$ is the direct contribution, and the second term is the resonant contribution with amplitude $R_{g}$, frequency $\omega_{0}$ and linewidth $\gamma$; q is the Fano factor which determines the asymmetrical shape. All the parameters are reported in Table 1. By changing the incident angle, we measure the different resonance peak frequencies and we can reconstruct the dispersion curve of the PhC surface states. The measured dispersion characteristic of the surface modes as a function of the in-plane wavenumber $k_{/ /}=n_{\text {prism }} k_{0} \sin \theta\left(k_{0}=2 \pi / \lambda_{0}\right)$ is reported in Figure 2.

By using a 3D FDTD approach, the mode dispersion at the top interface between the $\mathrm{PhC}$ slab and air, where the electric field is localized (Figure 3), has been determined. The dispersion curve calculated along the $\Gamma \mathrm{M}$ direction follows the trend of the surface plasmon wave dispersion (see the red curve in Figure 2). The good agreement between the experimental data and the simulated dispersion curve confirms the plasmon-like nature of the observed resonances.
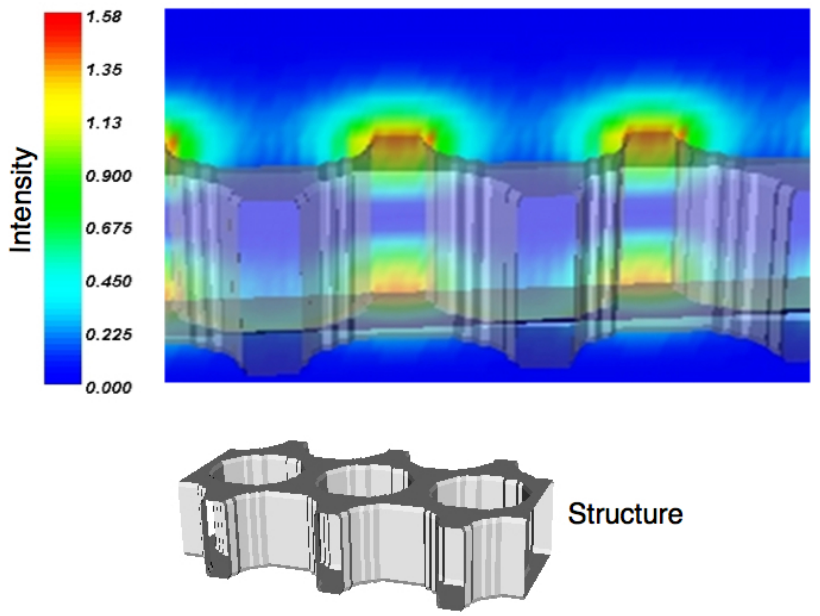

FIG. 3 Calculated field distribution in correspondence of a wavevector in M point of the Brillouin zone with two elementary cell of the $2 \mathrm{D} \mathrm{PhC}$ slab. The enhancement factor is about 80 .

\begin{tabular}{|c|c|c|c|}
\hline $\begin{array}{l}\theta \text { (deg) } \\
\pm 1\end{array}$ & $\begin{array}{l}\omega_{0}(a / \lambda) \\
\pm 2 \bullet 10^{-5}\end{array}$ & $\begin{array}{l}q \\
\pm 0.004\end{array}$ & $\begin{array}{l}\gamma(a / \lambda) \\
\pm 0.5\end{array}$ \\
\hline 57 & 0.31085 & 0.130 & 20.4 \\
\hline 58 & 0.31084 & 0.128 & 20.5 \\
\hline 59 & 0.31048 & 0.129 & 21.0 \\
\hline 60 & 0.31058 & 0.130 & 19.9 \\
\hline 61 & 0.31084 & 0.129 & 19.9 \\
\hline 62 & 0.31041 & 0.127 & 20.0 \\
\hline 63 & 0.31046 & 0.131 & 20.3 \\
\hline
\end{tabular}

TABLE 1 Parameter resulting from Fano fit. $\theta$ : incident angle; q: Fano factor; $\omega_{0}$ : resonance frequency; $\gamma$ : linewidth.

\subsection{Guided mode resonances}

By removing the prism, it is possible to detect and characterize guided mode resonance phenomenon in the same PhC structure. When incident radiation couples to a guided mode, the intensity of the reflected signal is reduced giving place to a very narrow GMR peak (see the inset in Figure 4). Also in this case we studied the reflected signal as function of the incidence angle. By varying the angle in the range from $40^{\circ}$ to $75^{\circ}$, chosen taking into account the laser tunability, the resonance wavelength shifts from $1531 \mathrm{~nm}$ to $1617 \mathrm{~nm}$. 


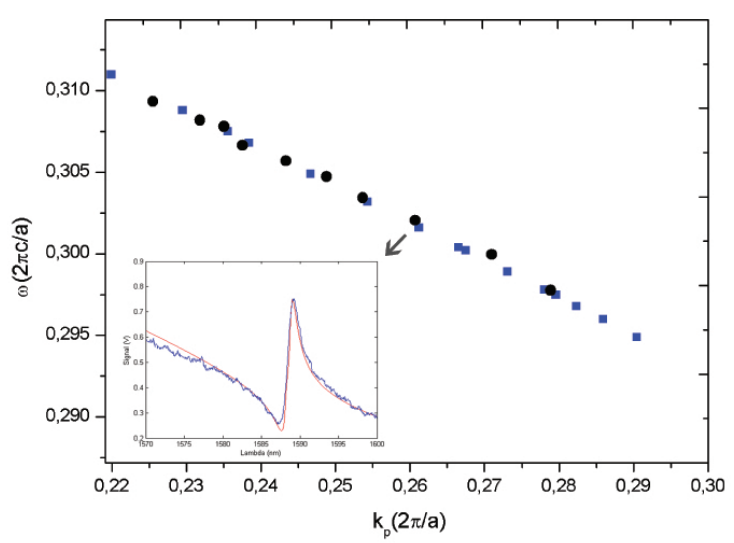

FIG. 4 Comparison between measured (black dots) and simulated (blue squares) dispersion characteristic of the $2 \mathrm{D} \mathrm{PhC}$ slab modes. The inset shows the experimental reflection signal at $\theta=65^{\circ}$ with the related best fit (red curve).

Infrared (IR) images of the radiation coupled into the structure in and out the resonance condition have been taken in front of the slab and on its lateral side (see Figure 5). In correspondence of a resonance $\left(\lambda=1588 \mathrm{~nm}\right.$ and $\left.\theta=65^{\circ}\right)$, the light propagates through the whole photonic crystal $\left(1 \times 1 \mathrm{~mm}^{2}\right)$ and then through the adjacent SOI planar waveguide (about $1 \mathrm{~mm}$ length) to be finally scattered at the edges of the SOI region by the irregularities of the lateral facet surface (see the inset in Figure 4). Out of resonance condition $(\lambda=1591 \mathrm{~nm})$, the radiation does not couple with the structure and the SOI edges are no more illuminated. The back-propagation of the coupled radiation is detected and this can be connected with negative index behavior of the photonic crystal under consideration. This effect is confirmed by placing an IR camera on the positive refraction side of the device: no radiation is detected.

By using RCWA, the time average Poynting vector is calculated. In resonance condition, the vector inverts direction in a few tens of nanometers while crossing the photonic crystal interface (Figure 6).

Finally, the experimental curve is fitted directly with Fresnel formula (2), considering the air/Lorentz resonator/Silicon Oxide three layer system. The results show that experimental values are in excellent agreement with theoretical calculations, see Figure 4 . Additionally, in the resonance condition $\left(\lambda_{0}=1588 \mathrm{~nm}\right.$ and $\left.\theta=65^{\circ}\right)$ the fit parameters are $\mathrm{f}=0.0007$, $\gamma=-1.5^{*} 10^{11} \mathrm{~s}, \varepsilon_{\infty}=12$ and they are constant for different incident angles, excepting for the resonant frequency $\omega_{0}$. In fact, by changing the angle of incidence, the band structure of the photonic crystal can be reconstructed [17, 19]. As reported in Figure 4, the measured dispersion (black dots) is in good agreement with the simulated dispersion points (blue squares) obtained by using RCWA. Moreover, this corresponds to the sign of the calculated and measured group velocity of the mode along the parallel wavevector, $\mathrm{k}_{/ /}[26]$.
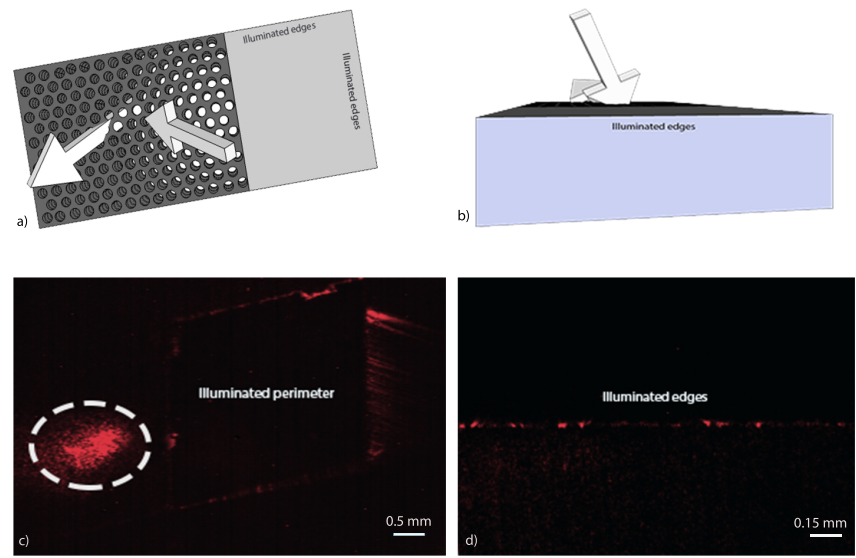

FIG. 5 Infrared images of the coupled radiation scattered from the Sol region (c,d) and sketches of the IR camera positions $(a, b)$. The circle indicates the spot laser incident on the sample.

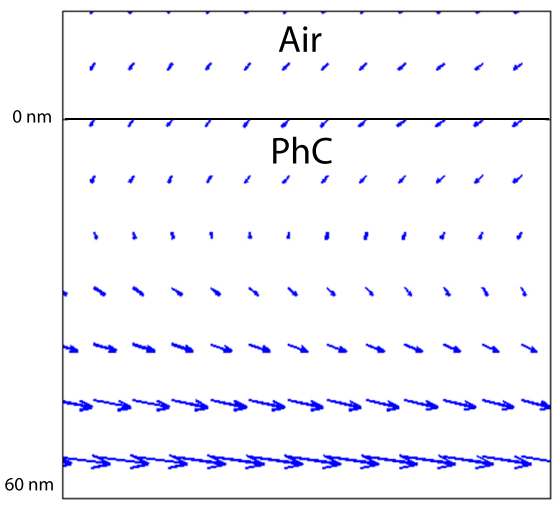

FIG. 6 The calculated time average Poynting vector in a $60 \times 60 \mathrm{~nm}^{2}$ area in the $\mathrm{xz}$ plane, crossing the PhC surface in the resonance condition.

\section{CONCLUSION}

In this paper the presence of two different resonance phenomena in a negative refractive photonic crystal is analyzed. At the interface air/PhC, electromagnetic surface states are demonstrated and characterized using the Kretschmann configuration. Additionally, the same structure shows a strong confinement of the light within the slab due to guided mode resonances coupling. Both optical phenomena, arising from a negative effective permittivity of the $\mathrm{PhC}$ are characterized by strong localization of laser light and narrow resonances in absence of absorption-losses. Therefore, the presented results open new solutions in light manipulation at nanoscale especially for sensing applications, avoiding the typical limitation of metallic plasmonic structure.

\section{ACKNOWLEDGEMENT}

Portions of this work were performed at the Molecular Foundry, Lawrence Berkeley National Laboratory, which is supported by the Office of Science, Office of Basic Energy 
Sciences, of the U.S. Department of Energy under Contract No. DE-AC02-05CH11231. ACDL was supported by an AIRC Start-up Grant 11454. ACDL and EDT were supported by a FIR project RBFR12WAPY of Italian Ministry of Education, Universities and Research.

\section{References}

[1] X. Guo, "Surface plasmon resonance based biosensor technique: A review," J. Biophotonics 5, 483 (2012).

[2] E. M. Larsson, J. Alegret, and D. S. Sutherland, "Sensing Characteristics of NIR Localized Surface Plasmon Resonances in Gold Nanorings for Application as Ultrasensitive Biosensors" Nano Lett. 7, 1256 (2007).

[3] P.-Y. Chung, T.-H. Lin, G. Schultz, C. Batich, and P. Jiang, "Nanopyramid surface plasmon resonance sensors" Appl. Phys. Lett. 96, 261108 (2010).

[4] T.-Y. Liu, K.-T. Tsai, H.-H.Wang, Y. Chen, Y.-H. Chen,Y.-C. Chao, H.-H. Chang, et al., "Functionalized arrays of Raman-enhancing nanoparticles for capture and culture-free analysis of bacteria in human blood," Nat. Commun. 2, 538 (2011).

[5] X. Yin, and L. Hesselink, “Goos-Hänchen shift surface plasmon resonance sensor," Appl. Phys. Lett. 89, 261108 (2006).

[6] R. Slavik, and J. Homola, "Ultrahigh resolution long range surface plasmon-based sensor," Sensor Actuat. B 123, 10 (2007).

[7] M. Nirschl, F. Reuter, and J. Vörös, "Review of Transducer Principles for Label-Free Biomolecular Interaction Analysis," Biosensors 1, 70 (2011).

[8] J. Kneipp, H. Kneipp, and K. Kneipp, "SERS- a single-molecule and nanoscale tool for bioanalytics," Chem. Rev. Soc. 37, 1052 (2008).

[9] J. Kneipp, H. Kneipp, M. McLaughlin, D. Brown, and K. Kneipp, "In Vivo Molecular Probing of Cellular Compartments with Gold Nanoparticles and Nanoaggregates," Nano Lett. 6, 2225 (2006).

[10] G. Rusciano, A. C. De Luca, G. Pesce, A. Sasso, G. Oliviero, J. Amato, N. Borbone, et al., "Label-Free Probing of G-Quadruplex Formation by Surface-Enhanced Raman Scattering," Anal. Chem. 83, 6849 (2011).

[11] G. Rusciano, A. C. De Luca, G. Pesce, and A. Sasso "On the interaction of nano-sized organic carbon particles with model lipid membranes," Carbon 47, 2950 (2009).

[12] R. Cush, J. M. Cronin, W. J. Stewart, C. H. Maule, J. Molloy, and N. J. Goddard, "The resonant mirror: a novel optical biosensor for direct sensing of biomolecular interactions Part I: Principle of operation and associated instrumentation," Biosens. Bioelectron. 8, 347 (1993).

[13] E. Descrovi, F. Frascella, B. Sciacca, F. Geobaldo, L. Dominici, and F. Michelotti, "Coupling of surface waves in highly defined onedimensional porous silicon photonic crystals for gas sensing applications," Appl. Phys. Lett. 91, 241109 (2007).

[14] M. L. Juan, M. Righini, and R. Quidant, "Plasmon nano-optical tweezers," Nat. Photonics 5, 349 (2011).

[15] K. Ishizaki, and S. Noda, "Manipulation of photons at the surface of three-dimensional photonic crystals," Nature 460, 367 (2009).

[16] E. De Tommasi, A. C. De Luca, S. Cabrini, I. Rendina, S. Romano, and V. Mocella, "Plasmon-like surface states in negative refractive index photonic crystals," Appl. Phys. Lett. 102, 081113, (2013).

[17] V. N. Astratov, D. M. Whittaker, I. S. Culshaw, R. M. Stevenson, M. S. Skolnick, T. F. Krauss, and R. M. De La Rue, "Photonic band- structure effects in the reflectivity of periodically patterned waveguides," Phys. Rev. B 60, 16255 (1999).

[18] V. N. Astratov, I. S. Culshaw, R. M. Stevenson, D. M. Whittaker, M. S. Skolnick, T. F. Krauss, and R. M. Rue, "Resonant Coupling of Near-Infrared Radiation to Photonic Band Structure Waveguides," J. Lightwave Technol. 17, 2050 (1999).

[19] V. N. Astratov, R. M. Stevenson, I. S. Culshaw, D. M. Whittaker, M. S. Skolnick, T. F. Krauss, and R. M. De La Rue, "Heavy photon dispersions in photonic crystal waveguides," Appl. Phys. Lett. 77, 178 (2000).

[20] S. Fan, and J. Joannopoulos, "Analysis of guided resonances in photonic crystal slabs," Phys. Rev. B 65, 2351121, (2002)

[21] S. Fan, W. Suh, and J. D. Joannopoulos, "Temporal coupled-mode theory for the Fano resonance in optical resonators," JOSA A 20, 569 (2003).

[22] J. Chen, Y. Wang, B. Jia, T. Geng, X. Li, L. Feng, W. Qian, et al., “Observation of the inverse Doppler effect in negative-index materials at optical frequencies," Nat. Photonics 5, 239 (2011).

[23] C. Luo, M. Ibanescu, S. G. Johnson, and J. D. Joannopoulos, "Cerenkov Radiation in Photonic Crystals," Science 299, 368 (2003).

[24] J. B. Pendry, "Negative Refraction Makes a Perfect Lens," Phys. Rev. Lett. 85, 3966 (2000).

[25] D. Lu, and Z. Liu, "Hyperlenses and metalenses for far-field superresolution imaging," Nat. Commun. 3, 1205 (2012).

[26] P. Dardano, M. Gagliardi, I. Rendina, S. Cabrini, and V. Mocella, "Ellipsometric determination of permittivity in a negative index photonic crystal metamaterial," Light: Sci. Appl. 1, e42 (2012).

[27] V. Mocella, S. Cabrini, A. Chang, P. Dardano, L. Moretti, I. Rendina, D. Olynick, et al., "Self-Collimation of Light over Millimeter-Scale Distance in a Quasi-Zero-Average-Index Metamaterial," Phys. Rev. Lett. 102, 133902 (2009).

[28] G. Di Caprio, P. Dardano, G. Coppola, S. Cabrini, and V. Mocella, "Digital holographic microscopy characterization of superdirective beam by metamaterial," Opt. Lett. 37, 1142 (2012).

[29] M. Notomi, "Theory of light propagation in strongly modulated photonic crystals: Refractionlike behavior in the vicinity of the photonic band gap," Phys. Rev. B 62, 10696 (2000).

[30] V. Mocella, P. Dardano, L. Moretti, and I. Rendina, "Influence of surface termination on negative reflection by photonic crystals," Opt. Express 15, 6605 (2007).

[31] M. Notomi, "Negative refraction in photonic crystals," Opt. Quant. Electron. 34, 133 (2002).

[32] V. Mocella, P. Dardano, I. Rendina, and S. Cabrini, "An extraordinary directive radiation based on optical antimatter at near infrared," Opt. Express 18, 25068 (2010).

[33] K. Vynck, D. Felbaca, E. Centeno, A. I. Cabuz, D. Cassagne, and B. Guizal, "All-dielectric rod-type metamaterials at optical frequencies," Phys. Rev. Lett. 102, 133901 (2009).

[34] M. Rybin, A. Khanikaev, M. Inoue, K. Samusev, M. Steel, G. Yushin, and M. Limonov, "Fano Resonance between Mie and Bragg Scattering in Photonic Crystals," Phys. Rev. Lett. 103, 023901 (2009).

[35] U. Fano, "The Theory of Anomalous Diffraction Gratings and of Quasi-Stationary Waves on Metallic Surfaces (Sommerfeld's Waves)," J. Opt. Soc. Am. 31, 213 (1941). 